\title{
Constraint on the Higgs-Boson Mass from Nuclear Scattering Data
}

\author{
J. A. Grifols, E. Massó, ${ }^{(a)}$ and S. Peris ${ }^{(b)}$ \\ Grup de Física Teórica, Universitat Autònoma de Barcelona, 08193 Bellaterra (Barcelona), Spain \\ (Received 30 June 1989)
}

\begin{abstract}
We exploit the experimental energy dependence of the neutron-lead scattering cross section to find a bound on the mass of the Higgs boson, $m_{H}$. We use the recently determined coupling constant of the Higgs particle to nucleons, $g=2.1 \times 10^{-3}$, and find $m_{H} \gtrsim 18 \mathrm{MeV}$.

PACS numbers: $14.80 . \mathrm{Gt}, 25.40 . \mathrm{Dn}$
\end{abstract}

The search for the Higgs boson is one of the most pressing issues in particle physics. A variety of physical processes have been used to constrain the mass of the Higgs particle. ${ }^{1}$ Some of them have theoretical uncertainties that make them not completely reliable at present. ${ }^{2}$ In the light of this fact it is important to study phenomena that may lead to safe constraints on the Higgs-boson mass.

Nuclear scattering data have proven to be useful for placing constraints on the Higgs boson. Barbieri and Ericson ${ }^{3}$ were the first to notice that the angular dependence of the scattering cross section of neutrons on a nucleus was sensitive to the potential originated by a lightboson exchange. Here we shall show that the consideration of the energy dependence of the total cross section also leads to a limit on the Higgs-boson mass, which turns out to be more stringent.

In the low-energy region the energy dependence of the neutron- $\mathrm{Pb}$ scattering cross section

$$
\sigma(k)=\sigma(0)+a_{1} k+a_{2} k^{2},
$$

with $k$ the wave vector of the incoming neutron, has been accurately determined by a recent experiment. ${ }^{4}$ The result is

$$
\begin{aligned}
& \sigma(0)=11.253(5) \mathrm{b}, \\
& a_{1}=0.60(51) \mathrm{bfm}, \\
& a_{2}=-371(27) \mathrm{bfm}^{2},
\end{aligned}
$$

where the fit is obtained with data in the range $1.5 \times 10^{-3} \leq k \leq 3.1 \times 10^{-2} \mathrm{fm}^{-1}$.

The aim of the experiment in Ref. 4 was the determination of the electric polarizability of the neutron, which is a parameter related to the linear term in $k$ in the cross section (1). This very small effect is largely due to the nuclear charge distribution and its electric field $^{4}$ and we will neglect it. On the contrary the values of $\sigma(0)$ and $a_{2}$ in Eq. (2c) can be understood in terms of a simple hard-core potential and its effective range. ${ }^{5}$

Indeed, the scattering amplitude for this nuclear potential reads

$$
f_{N}=-R\left[1-i k R-\frac{2}{3} k^{2} R^{2}+O\left(k^{3} R^{3}\right)\right],
$$

where $R$ is the radius of the potential and we have only displayed the relevant terms in the low-energy regime $(k R \ll 1)$. Defining the effective range $r_{\text {eff }}$ through

$$
\sigma=4 \pi R^{2}\left[1-R\left(R-r_{\mathrm{eff}}\right) k^{2}\right],
$$

then in our hard-core potential $r_{\text {eff }}$ is predicted to be

$$
r_{\mathrm{eff}}=\frac{2}{3} R \text {. }
$$

The consistency of this theoretical prediction with data can be seen as follows. In the presence of the nuclear potential only, the value of $R$ is obtained by using

$$
R=[\sigma(0) / 4 \pi]^{1 / 2}=9.46 \mathrm{fm} .
$$

Introducing this value in Eq. (5) we obtain

$$
r_{\mathrm{eff}}^{N}=6.3 \mathrm{fm} \text {. }
$$

This has to be compared to the effective range extracted from the experimental data in Eq. (2c),

$$
r_{\mathrm{eff}}(\operatorname{expt})=6.0 \pm 0.3 \mathrm{fm},
$$

which is in agreement with the prediction in Eq. (7).

A light Higgs boson would change the potential felt by a neutron when scattered off a nucleus. Indeed, a Higgs-boson exchange generates an attractive potential

$$
V_{H}=-\frac{A g^{2}}{4 \pi} \frac{e^{-m_{H} r}}{r}
$$

where $A$ is the atomic mass, $m_{H}$ the Higgs-boson mass, and $g$ the coupling constant of the Higgs boson to nucleons.

Now we need the scattering amplitude for the combined effect of the nuclear and the Higgs-induced potential, regarding the latter contribution as a perturbation. As we will see the Higgs-boson exchange only affects the value of the parameters $\sigma(0)$ and $a_{2}$ in Eq. (1). Therefore we will restrict our discussion to them. The proper treatment of our problem is the "distorted-wave Born approximation." 6 This approximation takes into account the distortion produced by the nuclear potential on the first-order scattering caused by $V_{H}$, which is the weak potential. The total scattering amplitude can be written 
as $^{6}$

$$
f=f_{N}+f_{H}+\delta f,
$$

where $f_{N}$ is the amplitude for the nuclear potential and is given by Eq. (3), and $f_{H}$ is the standard Bornapproximation amplitude for $V_{H}$ only,

$$
\begin{aligned}
f_{H}(\theta) & =2 \mu \frac{A g^{2}}{4 \pi} \frac{1}{2 k^{2}(1-\cos \theta)+m_{H}^{2}} \\
& \approx 2 \mu \frac{A g^{2}}{4 \pi m_{H}^{2}}\left[1-\frac{2 k^{2}}{m_{H}^{2}}(1-\cos \theta)+O\left(\frac{k^{4}}{m_{H}^{4}}\right)\right] .
\end{aligned}
$$

Here $\mu$ is the reduced mass and $\theta$ the scattering angle. The term $\delta f$ represents the distorting effect of the nuclear potential and is given by

$$
\delta f=-2 \mu \int_{0}^{\infty} V_{H}(r)\left[R_{0}^{2}(r)-j_{0}^{2}(k r)\right] r^{2} d r .
$$

In the integrand, we have the $S$-wave-function solution in the presence of the nuclear potential alone,

$$
R_{0}(r)=\theta(r-a) \frac{\sin k(r-a)}{k r},
$$

as well as the free solution $j_{0}(\rho)=(1 / \rho) \sin \rho$. Explicit calculations show that $\delta f$ is given by

$$
\delta f \approx \frac{\mu A g^{2}}{2 \pi}\left(I_{0}+k^{2} I_{1}\right)
$$

where

$$
I_{0} \equiv \int_{0}^{\infty} d r e^{-m_{H^{r}} r^{2}}\left(\frac{e^{-m_{H} R}}{r+R}-\frac{1}{r}\right),
$$

and

$$
I_{1} \equiv-\frac{1}{3} \int_{0}^{\infty} d r e^{-m_{H} r} r^{4}\left(\frac{e^{-m_{H} R}}{r+R}-\frac{1}{r}\right) .
$$

In the range $5 \lesssim m_{H} \lesssim 30 \mathrm{MeV}$, the above integrals can be very well approximated by

$$
\begin{aligned}
& m_{H}^{2} I_{0} \approx \frac{0.06}{\left(m_{H} R\right)^{1 / 2}}-0.68\left(m_{H} R\right)^{1 / 2}, \\
& m_{H}^{4} I_{1} \approx \frac{-0.1}{\left(m_{H} R\right)^{1 / 2}}+1.35\left(m_{H} R\right)^{1 / 2} .
\end{aligned}
$$

The final amplitude in Eq. (10) can now be calculated by adding $f_{H}$ and $\delta f$ given respectively by (11) and (14) to the nuclear amplitude $f_{N}$ given by (3).

Our idea is simple. It can be seen that the effect of the Higgs-boson potential is to increase the prediction for the effective range in Eq. (7), destroying the agreement we had in the limit $m_{H} \rightarrow \infty$, i.e., without Higgsboson exchange. To be conservative, we shall require that the nuclear and the Higgs-boson-induced potentials keep the agreement between theory and experiment within two standard deviations.

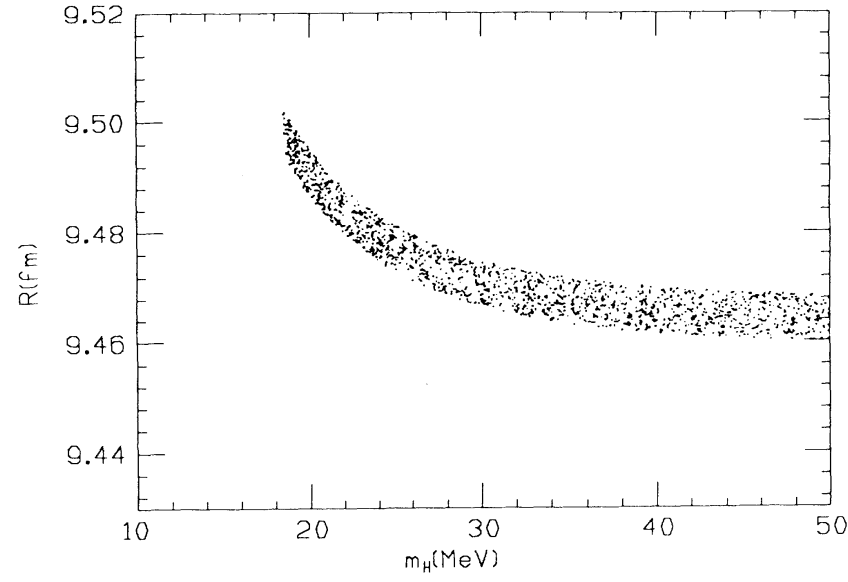

FIG. 1. Allowed values (shaded area) for $R$ and $m_{H}$.

The limit on $m_{H}$ obviously depends on the coupling $g$ of the Higgs boson on nucleons. Since the nucleon is a composite particle, some care is needed. The analysis of Ref. 7 leads to

$$
g=8.5 \times 10^{-4} \frac{n_{h}}{3},
$$

where $n_{h}$ is the number of heavy quark flavors. However, recent experimental evidence for a large strangequark sea in the nucleon increases the value in Eq. (19) for $n_{h}=3$ up to ${ }^{8}$

$$
g=2.1 \times 10^{-3} \text {. }
$$

We can now find our bound as follows. We evaluate the total contribution to $\sigma(0)$ and $a_{2}$ keeping $R$ and $m_{H}$ as free parameters. We require that the predicted values for $\sigma(0)$ and $a_{2}$ agree with the experimental data shown in Eqs. (2a) and (2c) at the two-standard-deviation level. The allowed values of $R$ and $m_{H}$ are shown in Fig. 1, for the $g$ given by Eq. (20). We see that for high masses of the Higgs boson, $R$ tends to the value in Eq. (6), as it should. Also, from Fig. 1 we can extract a lower limit on the mass of the Higgs boson,

$$
m_{H} \gtrsim 18 \mathrm{MeV} \text {. }
$$

This is our main result.

We have also calculated our limit for the coupling in Eq. (19). For $n_{h}=3$, we find

$$
m_{H} \gtrsim 12 \mathrm{MeV} \text {, }
$$

whereas for $n_{h}=4$ we get

$$
m_{H} \gtrsim 14 \mathrm{MeV} \text {. }
$$

Finally, it is interesting to compare our results with previous work. The method of Barbieri and Ericson ${ }^{3}$ leads to the limit $m_{H} \gtrsim 10 \mathrm{MeV}$ for $g=2.1 \times 10^{-3}$. $^{9}$ However, as the authors pointed out, their result holds if there are no fortuitous cancellations. Notice that our 
limits do not require this assumption.

Limits on $m_{H}$ coming from $K$ decays are subject to uncertainties $^{2}$ that make them unreliable at present. A strong bound can be obtained from $0^{+} \rightarrow 0^{+}$decays: ${ }^{10}$ $m_{H} \gtrsim 14 \mathrm{MeV}$ for the $g$ given by Eq. (20), and $m_{H} \gtrsim 9$ $\mathrm{MeV}$ when $g$ is the value of Eq. (19) with $n_{h}=3$. The other limits are weaker and we refer the reader to Refs. 1 and 11 for recent reports on them.

We thank Dr. J. Schmiedmayer for supplying us with useful information. This work was partially supported by research project Comisión Interministerial de Ciencia y Tecnologia.

(a) Address after September: Theory Division (CERN), 1211 Geneva 23, Switzerland.

(b) Present address: Theoretical Group, Department of Physics, University of California at Los Angeles (UCLA), Los Angeles, CA 90024-1547.

${ }^{1}$ For recent reviews, see R. N. Cahn, Rep. Prog. Phys. 52,
389 (1989); M. S. Chanowitz, Annu. Rev. Nucl. Part. Sci. 38, 323 (1988).

${ }^{2}$ S. Raby, G. B. West, and C. M. Hoffman, Phys. Rev. D 39, 828 (1989).

${ }^{3}$ R. Barbieri and T. E. O. Ericson, Phys. Lett. 57B, 270 (1975).

${ }^{4}$ J. Schmiedmayer, H. Rauch, and P. Riehs, Phys. Rev. Lett. 61, 1065 (1988).

${ }^{5} \mathrm{~J}$. Schmiedmayer (private communication).

${ }^{6}$ L. I. Schiff, Quantum Mechanics (McGraw-Hill, New York, 1968).

${ }^{7}$ M. A. Schifman, A. I. Vainshtein, and V. I. Zakharov, Phys. Lett. 78B, 443 (1978).

${ }^{8}$ T. P. Cheng, Phys. Rev. D 38, 2869 (1988); H. Y. Cheng, Phys. Lett. B 219, 347 (1989); R. Barbieri and G. Curci, Pisa University Report No. IFUP-TH 35/88 1988 (to be published).

${ }^{9}$ T. P. Cheng, Ref. 8.

${ }^{10}$ S. J. Freedman et al., Phys. Rev. Lett. 52, 240 (1984).

${ }^{11}$ H. Y. Cheng, Institute of Physics, Academia Sinica, Taipei, Report No. IP-ASTP-23-88, 1988 (to be published); in Proceedings of the Chinese-German Symposium on MediumEnergy Physics, Taipei, Taiwan, November 1988 (to be published). 